Review

\title{
The Gut-liver Axis in Immune Remodeling: New insight into Liver Diseases
}

\author{
Xinyu Yang1, ${ }^{12^{*}}$, Di Lu ${ }^{1,2^{*}}$, Jianyong Zhuo ${ }^{1,2}$, Zuyuan $\operatorname{Lin}^{1,2}$, Modan Yang1,2, Xiao Xu ${ }^{1,2 \bowtie}$ \\ 1. Department of Hepatobiliary and Pancreatic Surgery, the First Affiliated Hospital, Zhejiang University School of Medicine, 79 Qingchun Road, Hangzhou \\ 310003, China. \\ 2. NHFPC Key Laboratory of Combined Multi-Organ Transplantation, Hangzhou, 310003, China. \\ *These authors contributed equally to this work. \\ $\triangle$ Corresponding author: Prof. Xiao Xu, Division of Hepatobiliary and Pancreatic Surgery, Department of Surgery, First Affiliated Hospital, Zhejiang University \\ School of Medicine, 79 Qingchun Road, Hangzhou, 310003, China. E-mail address: zjxu@zju.edu.cn, Telephone No.: +86-571-87236601. \\ (c) The author(s). This is an open access article distributed under the terms of the Creative Commons Attribution License (https://creativecommons.org/licenses/by/4.0/). \\ See http://ivyspring.com/terms for full terms and conditions.
}

Received: 2020.03.27; Accepted: 2020.06.12; Published: 2020.06.23

\begin{abstract}
The gut microbiota consists of a dynamic multispecies community of bacteria, fungi, archaea, and protozoans, playing a fundamental role in the induction, training, and function of the host immune system. The liver is anatomically and physiologically linked to the gut microbiota via enterohepatic circulation, specifically receiving intestine-derived blood through the portal vein. The gut microbiota is crucial for maintaining immune homeostasis of the gut-liver axis. A shift in gut microbiota composition can result in activation of the mucosal immune response causing homeostasis imbalance. This imbalance results in translocation of bacteria and migration of immune cells to the liver, which is related to inflammation-mediated liver injury and tumor progression. In this review, we outline the role of the gut microbiota in modulating host immunity and summarize novel findings and recent advances in immune-based therapeutics associated with the gut-liver axis. Moving forward, a deep understanding of the microbiome-immune-liver axis will provide insight into the basic mechanisms of gut microbiota dysbiosis affecting liver diseases.
\end{abstract}

Key words: gut-liver axis; immunity; gut microbiome; dysbiosis; immunotherapy

\section{Introduction}

The reciprocal interaction of the gut-liver axis is established through the vascular route of the portal vein that directly transports gut-derived products to the liver, and the liver feedback route by which bile and antibodies travel to the intestine [1]. The intestinal barrier, a functional and anatomical structure consisting of intestinal mucosa and vascular endothelium, acts as a playground for the connections between the gut and the liver.

As an important constituent of the mucosal immune system, gut-associated lymphoid tissue (GALT) constructs a local immune environment that is both defensive and tolerant. The liver, as an organ linked to GALT, contributes to immune surveillance. [2] The liver, particularly enriched in innate immune cells, is a central immunological organ with high exposure to circulating antigens and endotoxins from the gut microbiota. [3] The dysregulation of the gut and liver immune system is involved with intestinal and liver diseases. [2] The intestinal mucosal surface forms a biophysical barrier, and mucus may enhance homeostasis by inducing immunoregulatory signals. For instance, MUC2 mucin has been found to imprint dendritic cells (DC)s tolerance after direct uptake [4, 5]. Intestinal epithelial cells (IECs) secrete conditioning cytokines, including thymic stromal lymphopoietin (TSLP) and transforming growth factor- $\beta$ (TGF- $\beta$ ) as well as prostaglandins (PGs) to prime DCs to promote the induction of $\mathrm{T}$ helper cell 17 (Th17) differentiation [6-10]. In addition, IECs also exert a strong influence on local IgA response by producing factors such as B-cell activating factor (BAFF, also known as TNFSF13B) and a proliferationinducing ligand (APRIL, also known as TNFSF13) 
[11]. In the lamina propria (LP), beneath IECs, both DCs and macrophages have specific adaptations promoting tolerance through the control of regulatory $\mathrm{T}$ cells (Tregs) and IgA+ B cells, which contribute to tolerance by displaying key gut-homing receptors CCR9 and a4 $\beta 7$ [12]. However, upon shifting to inflammation, T helper 1 (Th1) and Th17 responses are induced. Meanwhile, in the liver, the inflammatory activation of hepatic stellate and Kupffer cells can recruit innate immune effectors, including neutrophils, monocytes, natural killer (NK) cells and natural killer T (NKT) cells [3]. In addition, enterohepatic circulation of bile and blood carries products of digestion, along with immune molecules, antigens and microbial products; can also modulate intestinal immunity to some extent [13-15].

Here, a comprehensive review was conducted to illustrate the crosstalk between the gut microbiome and the host innate and adaptive immunity, highlighting the impact of gut microbiota dysbiosis on systematic immunity. In particular, the gut-liver axis involving the intestinal microbiome and hepatic immune system was outlined as a novel paradigm in immune-based therapies, on the basis of its vital role in the immune response.

\section{The crosstalk between the microbiome and immunity}

The immune system acts as a bridge to maintain the symbiotic relationship between the microbiome and the host. The gut microbiota modulates the host immune system to some extent, and the immune system inversely influences the composition of the gut microbiota. At the same time, owing to major changes induced by bacterial colonization of the intestinal tract [16], it is thought that the mucosal immune system is different from the systemic immune system, and is highly specialized and defined. In particular, it is thought that the mucosal immune system maintains gut homeostasis by promoting a beneficial microbiota composition, limiting the development of pathological processes and restricting microbial overgrowth [17].

The intestinal mucosa has a single cell layer of epithelial cells that separates the gut lumen harboring the commensal bacterial and foodborne pathogens from the body. In the mucosa-associated lymphoid tissue (MALT), GALT is composed of Peyer's patches (PPs) and various immune cells, such as antigenpresenting cells (APCs), innate lymphoid cells, and T and $B$ cells. The GALT serves as an essential component of the immune system and plays a critical role in systemic and local immune responses.

\section{Microbial immunomodulation in systemic immunity}

The microbiota actively shapes the host systemic immune response by mediating immune cell priming. DCs migrate to mesenteric lymph nodes (mLNs), where they present antigens to stimulate the production of Treg cells and effector $\mathrm{T}$ cells. These cells can balance gut tolerance and immunity by transmitting signals to the whole body, such as the production of regulatory cytokines (TGF- $\beta$, IL-10, and IL-35), and exerting the appropriate immunological reaction to combat specific pathogens by cross-reacting with similar epitopes [18-20]. The balance of beneficial bacteria versus pathogenic bacteria is referred to as "eubiosis", and is important in maintaining immunity. In contrast, when dysbiosis occurs (due to various causes, e.g., poor colonization, antibodies treatment or an unbalanced, unhealthy diet), the microbiota loses its anti-infectious potency against pathogenic bacteria. In addition, alterations in the microbiota under the new condition of dysbiosis can lead to a pathogenic tendency by producing opportunistic infections. For example, the induced alteration of the intestinal microbiota after antibiotic use could lead to metabolic disturbances, and therefore increase susceptibility to infections (e.g., fungal and Clostridium difficile infections) [21]. Gut dysbiosis may lead to a number of diseases, including gastrointestinal disorders, obesity, cardiovascular diseases, allergies and central nervous system-related diseases, through a series of alterations [22-24]. This alteration involves the disruption of the mucosal barrier, which impairs local immune responses. Given this intestinal dysbiosis, translocated bacteria and their derived products enter the peripheral circulation, thus influencing the systemic immunity through activating TLR signaling pathways and subsequently triggering a cascade of inflammatory cytokines [25]. During this process, the release of proinflammatory cytokines increases, changing the cytokine environment in the intestinal mucosa and mLNs into an inflammatory phenotype. Eventually, a deep inflammatory state is induced throughout the body [26].

\section{The microbiota in gut immunomodulation}

Local immunity is facilitated by pathogen recognition receptors (PRRs)-mediated recognition of pathogen-associated molecular patterns (PAMPs). PRRs, including TLRs on IECs and innate immune effectors in the gut, are a class of germlineencoded receptors that recognize PAMPs. The activation of PRRs is crucial for the initiation of innate immunity, which plays a key role in the first-line of 
defense until more specific adaptive immunity is developed.

Furthermore, microbiota-derived metabolites, including short-chain fatty acids (SCFAs), can also modulate local immune responses. SCFAs exert strong epigenetic regulatory effects on B cell differentiation by promoting the production of both IgA and IgG isotypes [27-29]. SCFAs can also upregulate three important metabolic processes, glycolysis, oxidative phosphorylation and lipogenesis in B-cells, which are necessary to produce cellular building blocks and energy to support plasma B-cell differentiation [30]. mLNs are sites in which commensal bacteria transform adaptive immune responses, mainly by promoting the differentiation of naive $T$ cells [31]. Once DCs become mature, they migrate to $\mathrm{mLNs}$, transforming naive $\mathrm{T}$ cells into $\mathrm{CD}^{+}$Tregs and Th17 cells, which possess the ability of modulating intestinal immune balance [32, 33]. Tregs have the ability to induce mucosal tolerance and produce of immunosuppressive cytokines (e.g., IL-10). Of note, continuous crosstalk occurs between intestinal symbionts and mucosal T cells (e.g., Tregs) because bacterial metabolites such as SCFAs promote the maintenance of $\mathrm{T}$ cells in the intestine. The function of SCFAs relies on their capacity to suppress histone deacetylase (HDAC) activity, indicating the presence of epigenetic regulation [34]. In detail, the major components of SCFAs, including propionate and butyrate, can inhibit the HDAC 1 and 3. HDACs and histone acetylase (HATs) induce the histone acetylation, which is critically important epigenetic mechanisms involved in the regulation of gene expression by serving as a switch between permissive (via HAT-induced acetylation) and repressive chromatin (through HDAC-driven deacetylation). Butyrate also seems to influence PAMPs-induced inflammatory state, a previous study has found that butyrate inhibits peptidoglycan-induced TNF-a and IL-1 $\beta$ expression in THP-1 cells [35].

Resident microbiota contributes to the coordination of Treg/Th17 axis and safeguarding the mucosa. Microbiota dependent TLR signaling is involved in the regulation of inflammation and tolerance. TLR2/MyD88signaling is required for generation and expansion of Nrp1 $1^{\text {low Foxp } 3^{+} \text {Tregs }}$ and Treg17 cells in oral and gut mucosa. [36] The capsular polysaccharide A of the Bacteroides fragilis can promote the production of IL-10 by Foxp3 $3^{+}$Tregs in a TLR2 dependent manner, thus facilitating the mucosal tolerance [37]. The presence of commensal bacteria is required for the induction of steady-state Th17 cells in the intestinal lamina propria. In germ- free (GF) mice, Th17 cells are significantly decreased, but they can be induced by segmented filamentous bacteria $[38,39]$. Th17 cells in the lamina propria of the gut play a critical role in preventing pathogen infection. Modulation of Th17 cells is currently viewed as a potentially pharmacological target. Inhibition of a Th17 response would result in downregulation of pro-inflammatory IL-17 production [40].

Furthermore, Th2 immune responses contribute to the maintenance of mucosal homeostasis through increased secretion of IL-4, IL-5, IL-9, IL-13 and IL-21, which confer protection against helminthic infection [41]. The "core" signature of Th2 responses is the secretion of the cytokines IL-4, IL-5, and IL-13 by lymphocytes that express transcription factors, such as GATA binding protein-3, STAT-5, and STAT-6 [42]. A healthy balance of Th1/Th2 cells is essential for immune regulation. The gut microbiota and its metabolites influence the balance of Th1/Th2 cells ratio in the intestinal tract. Colonization of GF mice with Bacteroides fragilis was found to be sufficient to correct an imbalance between Th1 and Th2 cells [43]. Recent research has also indicated that yeast $\beta$-glucan, a polysaccharide of the gut microbiota, can contribute to the differentiation and secretion of Th2 cells by elevating the expression of GATA3 mRNA [44].

The interaction between microbiota and immunity also depend on the physiological location. With an oxidative stress sensitive (Ox-S)/oxidative stress-resistant $(\mathrm{Ox}-\mathrm{R})$ bacterial ratio increase, the colonic microbiota-immunity interaction is different from that in the small intestine in terms of oxygen tolerance [45]. Production of SCFAs, especially butyrate, in the gut microbiome is required for maturation of the gut microbiota [46]. Butyrate is produced from acetate and lactate by the Ox-S gut microbiota, mainly Lachnospiraceae, Ruminococcaceae and Bacteroidetes [47]. In microbiota-immunity interactions, on the one hand, butyrate downregulates gut mucosal immunity with an increase in FoxP3 ${ }^{+}$ Tregs in the colon. On the other hand, butyrate upregulates antigen-specific immune-response induction through decreased NKp46 group 3 innate lymphoid cells (ILC3s) in the Peyer's patches of the terminal ileum $[48,49]$. Therefore, butyrate modulates gut mucosal immunity depending on the physiological location, with induction of antigenspecific immune responses in terminal ileal PPs, but immunological tolerance within the colon. Different immune regulations between the terminal ileum and the colon play a vital role in immunosurveillance and anaerobic biological processes for host health. 


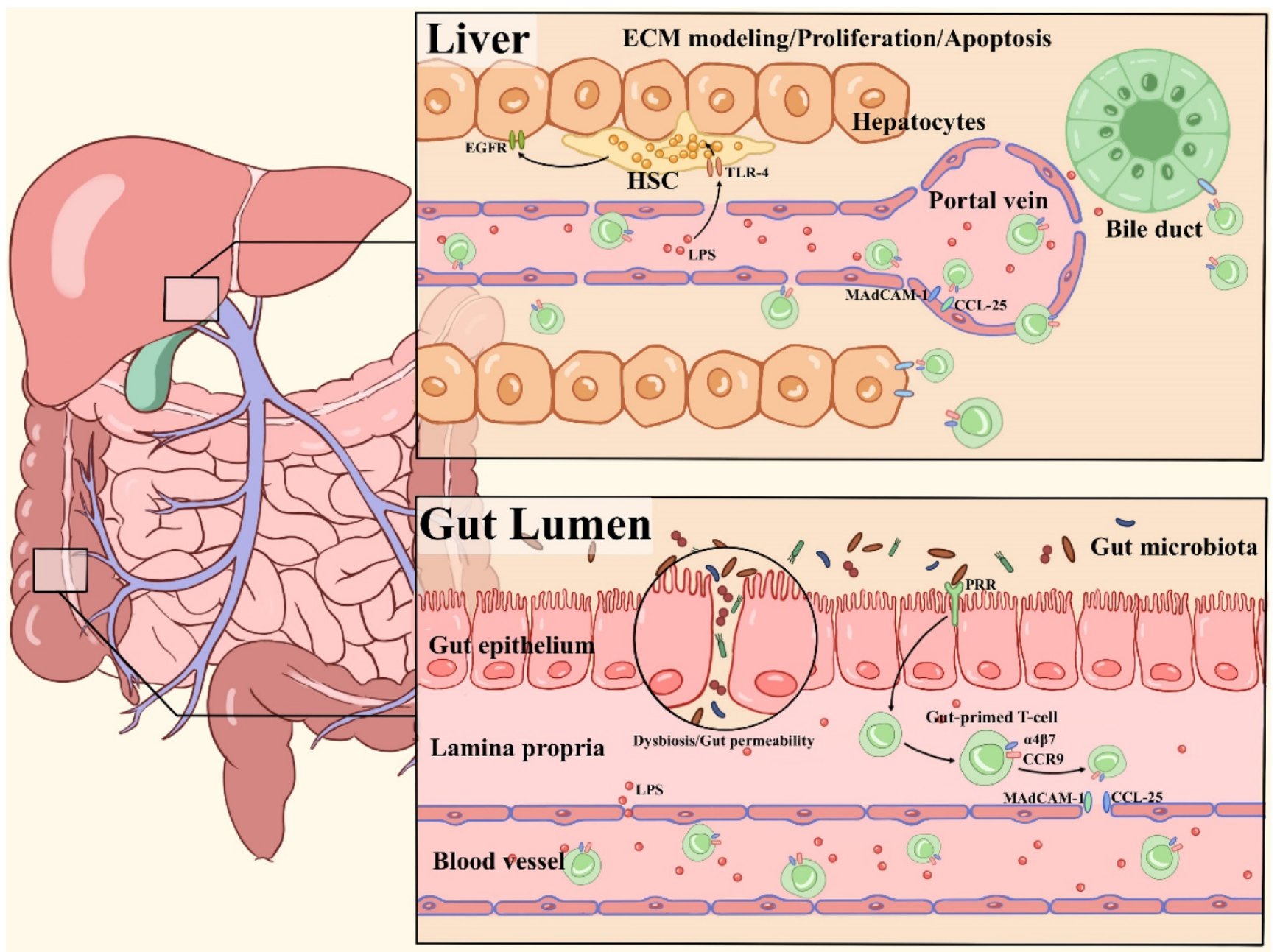

Figure 1. Overview of major pathways of the gut microbiota in shaping hepatic immunity. (1) Chronic inflammatory liver diseases are associated with gut microbiota dysbiosis, intestinal permeability changes, and MAMPs (LPS) translocation to the liver. TLR4 signaling is activated by LPS in hepatic stellate cells (HSCs) and hepatocytes, leading to extracellular matrix (ECM) remodeling, secretion of proinflammatory cytokines and activation of epidermal growth factor (EGF) family members, which influence the proliferation and apoptosis of hepatocytes. (2) Pathogens derived from bacterial translocation from the inflamed gut to the portal circulation due to increased intestinal permeability, driving the local inflammation via PRR activation. The naive T cells are imprinted with the gut-homing receptors integrin $\alpha 4 \beta 7$ and CC-chemokine receptor 9 (CCR9), these so-called gut-primed T cells will recirculate into the liver via venules by binding to CCL25 and MAdCAM-1 on hepatic endothelial cells. Then, chemokines secreted by epithelial target cells (hepatocytes or biliary epithelial cell) are in response to the activation of chemokine receptors such as CCR6 on effector cells. As a result, chronic inflammation, immune attack and destruction of bile ducts occur.

\section{The gut microbiota in shaping hepatic immunity}

The liver is continuously exposed to an overload of antigenic stimuli which includes pathogens and endotoxins from the gut microbiota, and plays a critical role in maintaining immunological tolerance [50-52]. The liver is considered a unique immunological organ with a predominantly innate immune role, as it contains an unusually large number of innate immune cells, including NK cells, NKT cells, macrophages and $\gamma \delta \mathrm{T}$ cells [53]. A previous report demonstrated the inflammasome-IL18 regulatory signaling circuit impacted maturation of hepatic NK cells, surface expression of the death ligand FasL, and capacity to kill FasL sensitive tumors. This study defines a regulatory circuitry in the innate immune system that links microbiota- derived Nlrp3 inflammasome activation by endogenous IL-18 signal to effective hepatic NK cellmediated tumor attack [54]. The microbiota also sustains the hepatic IL-17A-producing $\gamma \delta \mathrm{T}$ ( $\gamma \delta \mathrm{T}-17)$ cell homeostasis, including activation, survival and proliferation. Li et al. showed that colonization with $E$. coli induces generation of $\gamma \delta \mathrm{T}-17$ cells in a dose-dependent manner [55]. Gut bacteria shed microbial-associated molecule patterns (MAMPs), such as lipopolysaccharide (LPS) and endotoxin, into the portal venous circulation. The molecules can affect the Kupffer cell phenotype through TLR ligands and trigger a subsequent adaptive immune response via Kupffer cell-derived pro-inflammatory cytokines, thus shaping liver immunity $[56,57]$. Here, we discuss in detail two main pathways by which the gut microbiota shapes hepatic immune cell responses to modulate liver-associated diseases (Figure 1). 
Microbial translocation and immune activation in the liver

The intestinal mucosal immune system is comprised of specialized structures and sites, including PPs, lymph nodes, the lamina propria and the epithelium, that contain a variety of cells that participate in continuous activation, migration and homing [58]. Among them, the gut-draining mLNs are critical sites for orchestrating adaptive immunity. To support the failing intestinal barrier, the liver acts as a second firewall, filtering bacteria that drain from the intestine into the hepatic portal vein $[59,60]$. Pattern recognition receptors (PRRs) function as sensors of MAMPs, such as LPS, lipoteichoic acid (LTA), peptidoglycan and lipoproteins [61]. Once MAMPs arrive to the liver through the portal vein, they can activate innate immune cells expressing PRRs, including Kupffer cells, hepatic sinusoidal endothelial cells (HSECs) and biliary epithelial cells, via PRRs binding (e.g., Toll-like receptor 4) and induce inflammation [62-64]. For example, the LPS/TLR4 pathway upregulates the epiregulin hepatomitogen, an epidermal growth factor (EGF) family member in HSECs, leading to EGFR and HER2 activation, whereas it inhibits hepatocyte apoptosis during the late stages of hepatocarcinogenesis [65, 66]. Increased gut-derived MAMPs shift to the liver during dysbiosis and subsequently shape the hepatic immune milieu by regulating inflammatory cytokines. LPS/TLR4 activation in Kupffer cells induces the secretion of pro-inflammatory cytokines, such as TNF-a and IL-6. These elevated cytokines enhance the permeability of the hepatic sinus and the proliferation of hepatocytes, resulting in increased aggressiveness of hepatocellular carcinoma (HCC) [67]. In addition, as IL-6 is an activator of the JAK-STAT signaling pathway, its upregulation can lead to the polarization of M2 macrophages, potentially contributing to HCC metastasis and drug resistance in chemotherapy [68]. In summary, bacterial translocation might drive excessive immune responses that may compromise the health of the host. Liver cells, especially hepatocytes and cholangiocytes, are particularly susceptible to changes in the immune milieu. The 'leaky gut' hypothesis also links translocating gut microbial products with the onset and progression of nonalcoholic fatty liver disease (NAFLD) and alcohol-related liver disease (ALD), and for a long time, they were considered one of its major contributors. Compared with healthy individuals, patients with NAFLD were shown to have increased intestinal permeability and tight junction alterations [69]. In addition, chronic alcohol abuse results in a disruption of the intestinal barrier, related to the development and progression of ALD [70].

\section{Recruitment of mucosal lymphocytes into the liver}

In parallel to the 'leaky gut' hypothesis, a 'gut lymphocyte homing' hypothesis has been adopted. It studies the reciprocal interaction between the mucosal immune system and hepatic immunity through the gut-liver axis. The adhesion of lymphocytes into the liver differs from the classical migration pathway as described earlier [71, 72]. Among them, there is an important role for lymphocyte adhesion molecules expressed by HSECs, including vascular cell adhesion molecule-1 (VCAM-1), intercellular adhesion molecule-1 (ICAM-1) and common lymphatic endothelial and vascular endothelial receptor-1 (CLEVER-1) [73]. Hepatic VCAM-1 is only weakly expressed on human portal ECs, but its expression is increased by inflammatory cytokines, and it therefore contributes to homing through both the portal veins and hepatic sinusoids [74, 75]. Interestingly, in an antigen-driven mouse model of biliary injury, VCAM-1-mediated adhesion of a4 $\beta 1$-positive hepatic $\mathrm{T}$ cells to cholangiocytes reduced apoptosis, thus promoting $\mathrm{T}$ cell survival and continuance of hepatic inflammation [76]. The hepatic endothelium has been shown to aberrantly express the gut-specific chemokine CCL25 and recruit gut-homing CCR9+ lymphocytes by binding to mucosal addressin cell adhesion molecule-1 (MAdCAM-1) [77]. These results indicate that gut-primed $\mathrm{T}$ cells migrate from the gut to the liver and induce immune responses in the liver [78]. The pathogenesis of primary sclerosing cholangitis (PSC) has been suggested to be related to inflammatory bowel disease (IBD) and inflammation in the portal tract. The 'leaky gut' and 'gut lymphocyte homing' hypothesis explain the correlation observed between IBD and PSC [79]. These phenomenons highlight the association of the gut-liver axis in these immune disorders.

\section{The microbiota-immune interaction in liver diseases}

Since the portal vein provides approximately $70 \%$ of the liver's blood supply, dysbiosis of the gut microbiota can therefore shape immune cell responses in the liver and is related to various liver diseases including NAFLD and ALD [80-85]. Salzman et al. believed that the negative effects of gut dysbiosis are accompanied by gut microbiota-mediated inflammation of the local mucosa that encourages mucosal immune dysfunction, thus contributing to NAFLD pathogenesis [86]. It is documented that certain gut microbiota members, including members of the Bifidobacterium genus, influence Treg development, whereas others, such as segmented filamentous bacteria (SFB), promote Th17 development [87-89]. These particular members of the 
microbiota are associated with liver diseases, along with immune-related biological processes, including activation of innate and adaptive immune responses, suppression of inflammatory cytokine production and inhibition of immune cell recruitment [90].

\section{The immune shift under the treatment of liver diseases: the role of the gut-liver axis}

Mounting evidence highlights the role of the commensal microbiome in influencing the immune milieu of liver diseases, which, in turn, suggests the potential therapeutic utility of regulating the immune response via multiple microbiome manipulation methods, such as antibiotics, probiotics, prebiotics, fecal microbiome transplant (FMT), diet regulation, and administration of bacterial consortia. Efforts are currently underway to produce or enhance therapeutic responses by influencing the immune milieu-associated with the gut-liver axis. Several studies have presented a preliminary benefit in malignant and nonmalignant liver diseases.

\section{The gut microbiota and autoimmune liver diseases}

PSC is the most common autoimmune liver disease, characterized by a progressive immunemediated liver damage that leads to fibrosis of the biliary tree with chronic cholestasis and often endstage liver cirrhosis [91]. PSC is often associated with IBD, because colonic inflammation can lead to increased intestinal epithelium permeability and bacterial translocation to the liver, accelerating activated $\mathrm{T}$ cell migration from the intestine to the liver triggering immune-mediated damage [92]. Recently, a study has shed light on this association, showing that Klebsiella pneumoniae (K. pneumoniae) can disrupt gut barrier integrity and then trigger innate immune responses in the liver following translocation [93]. Using gnotobiotic mice and bacterial-organoid co-culture system, researchers demonstrated that PSC-derived K. pneumoniae was related to bacterial translocation and susceptibility to Th17-mediated hepatobiliary injuries. These results indicate that disease-specific bacteria might serve as a potential therapeutic target for PSC.

\section{The gut microbiota and cancer immunotherapy}

Several studies have reported that immunotherapy responders have differential gut microbiota signatures than nonresponders, and these specific signatures are related to enhanced systemic immunity and increased intratumoral immune infiltration. Recently, reports found that responder and nonresponder phenotypes could be replicated in antibiotic-treated or germ-free mouse models through fecal microbiota transplant (FMT). This phenomenon implied that therapeutic responses can be regulated through the modulation of the gut microbiota [94-97]. As shown in Table 1, some clinical trials were also conducted to explore the value of the gut microbiota in improving immunotherapy effects. In addition, a study reported that cyclophosphamide (an immunostimulatory agent) alters the composition of the natural microbiota in the small intestine of mice and facilitates a shift of selected Gram-positive bacteria to secondary lymphoid organs. These bacteria stimulate the generation of a specific subset of "pathogenic" Th17 cells and the memory Th1 cell immune response. These cells enhance the therapeutic effect of cyclophosphamide by expressing IL-17. This conversion into IL-17-producing cells was not observed in the absence of the gut microbiota [98]. The efficacy of immune checkpoint inhibitors (ICIs), including anti-PD-1/PD-L1 and anti-CTLA-4 agents, may be affected by the components of the gut microbiota. A recent study found that bacterial species, including Bifidobacterium longum, Collinsella aerofaciens, and Enterococcus faecium, were more abundant in anti-PD-L1 therapy responders than in the nonresponders. Remodeling the gut microbiota of germ-free mice with fecal material obtained from patients who responded to anti-PD-L1 agents could enhance $\mathrm{T}$ cell responses and improve the efficacy of anti-PD-L1 therapy [97]. Hepatocellular carcinoma (HCC) and cholangiocarcinoma (CCA) are the most common histological types of liver cancer. The gut microbiota is also associated with the response to anti-PD-1 immunotherapy in HCC patients. Through metagenomic sequencing, a study reported that fecal samples from HCC patients responding to anti-PD-1 immunotherapy showed higher taxa richness and more gene counts than those from nonresponders. These results highlight an important role of microbiota in disease monitoring and treatment decision-making [99]. Fundamental research also revealed that microbiome-induced innate immune change has an impact on the antitumor immune response in liver tumors. Using mouse models of primary liver tumors and metastatic liver tumors, Ma and colleagues found that Clostridium species could inhibit the recruitment of hepatic NKT cells and thereafter suppress antitumor immunity in the liver, against both primary and metastatic liver tumors. In addition, antibiotic treatment was shown to alter the composition of the gut microbiota and inhibit tumor growth [100]. In a summary, these treatment-responsive microbiome signatures suggest high potential for identifying novel combinations with checkpoint inhibitors. However, numerous 
issues remain to be addressed regarding the microbial product administered.

\section{The impact of gut dysbiosis on ischemia reperfusion injury (IRI) and immune-mediated allograft injury}

Both animal and human studies have revealed that the gut-liver axis acts as an important modulator in allograft innate and adaptive immunity, implicating the therapeutic effect of microbiota-based treatment in immune-mediated allograft injury [101, 102]. IRI causes organ dysfunction and failure after liver surgery and represents a major risk factor for the development of both acute and chronic graft rejection in liver transplantation (LT) [103]. Importantly, it is the limiting factor in the utility of marginal or extended criteria donor organs, which are highly susceptible to IRI, contributing to severe organ shortages. IRI is a dynamic process in which innate and adaptive immune inflammatory responses play an essential role in developing early allograft dysfunction (EAD) or primary nonfunction (PNF) $[104,105]$. The impact of the gut microbiota on early innate immune activation during IRI has been reported. A protective effect was found in a mouse IRI model by administrating probiotics, mainly Bifidobacterium and Lactobacillus [106]. Furthermore, in a rat model of LT, liver ischemic preconditioning not only improved hepatic graft function and intestinal barrier function, but also promoted restoration of the intestinal microbiota following LT, especially increasing Lactobacillus, Bifidobacterium, and Clostridiales. This process may further benefit hepatic grafts via positive feedback of the gut-liver axis [107]. Microbiota-derived SCFAs produced by the fermentation of nondigestible fiber can enable communications between the microbiome and host tissues and act as critical modulators in liver immune homeostasis. Intravenous administration of butyrate, a four-carbon fatty acid, can alleviate IRI-induced liver injury by suppressing inflammatory factor production and preventing NF- $\mathrm{KB}$ activation in
Kupffer cells [104, 108].

\section{The gut microbiota in other liver diseases - emerging indications}

The gut microbiota is also involved in the immunoregulation of viral, alcoholic and drug-related liver diseases. In chronic hepatitis B virus (HBV) infection, other than being directly caused by the adaptive immune response, liver injury is also indirectly caused by the innate immune response through gut microbiota-produced PAMPs [109]. TLRs are the main pattern recognition receptors in the innate immune system and play a vital role in the immune response [110]. The gut microbiota plays critical roles in drug metabolism. Individual variations in the gut microbiota contribute to the interindividual differences in response to drug therapy, including differences in drug-induced toxicity and efficacy [111]. Microbiota-derived metabolites can indirectly affect xenobiotic metabolism pathways. A previous study found that the relative abundance of Mucispirillum, Turicibacter and Ruminococcus before acetaminophen (APAP) dosing was correlated with increased hepatotoxicity, indicating APAP-induced acute liver injury [112]. In addition, gut dysbiosis is also associated with immune dysregulation during the onset and progression of alcohol-related liver disease (ALD). Researchers found that intestinal deficiency in two antimicrobial proteins, regenerating islet derived (Reg)-3b and Reg3g, can promote the progression of ethanol-induced fatty liver disease toward steatohepatitis $[113,114]$. The intestinal mucus layer is composed of mucins, predominantly MUC2, secreted by goblet cells of the intestine. Muc2 $\%$ mice are protected from intestinal bacterial overgrowth and dysbiosis in response to alcohol feeding [115]. Enterococcus faecalis (E. faecalis) is related with the progression of ALD, and a recent study indicated that bacteriophages can attenuate ALD through specifically targeting the cytolytic E. faecalis $[116,117]$.

Table 1. Clinical trials aiming to improve cancer immunotherapy by modulating the gut microbiota

\begin{tabular}{|c|c|c|c|c|c|c|}
\hline Registration number & Cancer type & $\mathrm{n}$ & Objective & Intervention & Outcome measure(s) & Country \\
\hline NCT03358511 & Breast cancer & 20 & $\begin{array}{l}\text { To assess the impact of presurgical } \\
\text { probiotics on antitumor immune } \\
\text { function }\end{array}$ & $\begin{array}{l}\text { Primal Defense Ultra }{ }^{\circledR} \text { probiotic } \\
\text { formula. }\end{array}$ & $\begin{array}{l}\text { Mean number of cytotoxic T } \\
\text { lymphocytes (CD8 }{ }^{+} \text {cells). }\end{array}$ & USA \\
\hline NCT03772899 & Melanoma & 20 & $\begin{array}{l}\text { To assess the safety of combining FMT } \\
\text { and immunotherapy in advanced } \\
\text { melanoma patients }\end{array}$ & $\begin{array}{l}\text { FMT combined with approved } \\
\text { immunotherapy (either } \\
\text { pembrolizumab or nivolumab). }\end{array}$ & Adverse effect assessments. & Canada \\
\hline NCT04130763 & $\begin{array}{l}\text { Gastrointestinal } \\
\text { system cancer }\end{array}$ & 5 & $\begin{array}{l}\text { To study the use of FMT in patients } \\
\text { with gastrointestinal system cancer for } \\
\text { whom anti-PD- } 1 \text { treatment failed }\end{array}$ & $\begin{array}{l}\text { FMT capsule produced by the } \\
\text { gut microbiota of these healthy } \\
\text { people. }\end{array}$ & $\begin{array}{l}\text { ORR; the safety of FMT } \\
\text { capsule was assessed by } \\
\text { adverse events. }\end{array}$ & China \\
\hline NCT03341143 & Melanoma & 20 & $\begin{array}{l}\text { To study the concurrent use of FMT } \\
\text { with pembrolizumab in patients with } \\
\text { anti-PD-1 agent-resistant/refractory } \\
\text { melanoma }\end{array}$ & $\begin{array}{l}\text { FMT combined with } \\
\text { Pembrolizumab. }\end{array}$ & $\begin{array}{l}\text { ORR; alterations in T cell } \\
\text { composition and function; } \\
\text { alterations in innate/adaptive } \\
\text { immune system subsets. }\end{array}$ & USA \\
\hline
\end{tabular}




\section{Conclusions and future trends}

The gut microbiota is a central participant in regulating hepatic immunity through the gut-liver axis, which refers to the reciprocal interaction that takes place between both the gut and its microbiota, and the gut and the liver on the other. Furthermore, there is growing evidence that dysregulation of gut-liver immunity leads to the progression of liver diseases, including malignant tumors. Thus, the mechanisms by which innate and adaptive immunity are influenced through the gut-liver axis have become attractive research topics.

Elucidation of the detailed immune changes associated with the gut microbiota induced by the gut-liver axis may contribute to the development of promising therapeutic strategies for liver diseases. A liver cancer-specific gut microbiota and the immune response induced by gut microbial species might be uncovered in the near future. Likewise, microbialbased interventions have demonstrated a benefit in improving allograft function and reducing the risk of post-LT complications, implicating that microbiotabased therapies will be utilized widely to improve clinical outcomes in post-LT patients. Currently, preclinical studies have demonstrated the bidirectional effect of the gut microbiota on the response to immunotherapy in mouse models. However, the biological mechanisms at cellular and molecular levels underlying the relation between gut microbiota and positive response to ICIs need to be further elucidated. In addition, whether the gut microbiome as a whole, or specific bacteria, can influence therapeutic responsiveness, and which specific composition is the most ideal for promoting cancer immunotherapy are still unclear. Therefore, thoroughly studying the multiplicity of therapeutic options, such as diet modification and FMT, is required in future clinical research. With $16 \mathrm{~S}$ rRNA gene-based microbial profiling technology, we can better identify the composition of the gut microbiota. Moreover, a comprehensive characterization at the species level can further promote our understanding of the effects of the gut microbiome on gut-liver immunity, thus allowing microbiome modulation to enhance the efficacy of immunotherapy in liver diseases. Moreover, advanced approaches such as clustered regularly interspaced short palindromic repeat (CRISPR)-based technologies have revolutionized the genome editing field and have already been applied to the development of novel antimicrobial strategies [118, 119]. With specific genetic properties, selective and efficient eradication of pathogenic bacteria has become a reality. Though still rather early in clinical application, these emerging technologies indicate exciting possibilities for microbiota modulation in the future.

In conclusion, it is still unclear which specific composition of the gut microbiome is most conducive to promoting a beneficial immune response. There are a variety of treatments that alter the microbiome, which require a future careful testing in the setting of clinical trials. Only by fully understanding these interactions we can learn to optimally target the microbiota to prevent and alleviate liver diseases via the remodeling of the immune milieu between the gut and the liver.

\section{Acknowledgements}

We thank Ms. Cen for technical assistance and secretarial work.

\section{Funding Support}

This work was supported by the State Key Program of National Natural Science Foundation of China under Grant No. 81930016; the National Major Scientific and Technological Special Project under Grant No. 2017ZX10203205; and National Natural Science Funds for Distinguished Young Scholar of China under Grant No. 81625003.

\section{Competing Interests}

The authors have declared that no competing interest exists.

\section{References}

1. Albillos A, Gottardi A, Rescigno M. The gut-liver axis in liver disease: pathophysiological basis for therapy. Journal of hepatology. 2019.

2. Trivedi PJ, Adams DH. Gut-liver immunity. Journal of hepatology. 2016; 64: 1187-9.

3. Heymann F, Tacke F. Immunology in the liver--from homeostasis to disease. Nature reviews Gastroenterology \& hepatology. 2016; 13: 88-110.

4. Shan M, Gentile M, Yeiser JR, Walland AC, Bornstein VU, Chen K, et al. Mucus enhances gut homeostasis and oral tolerance by delivering immunoregulatory signals. Science (New York, NY). 2013; 342: 447-53.

5. MS, M G, JR Y, AC W, VU B, K C, et al. Mucus enhances gut homeostasis and oral tolerance by delivering immunoregulatory signals. Science (New York, NY). 2013; 342: 447-53

6. GS A, K L, W J, G L, AB K, T G-W, et al. Secretory leukocyte protease inhibitor mediates non-redundant functions necessary for normal wound healing. Nature medicine. 2000; 6: 1147-53

7. P K, PL V, JH S, EC dJ, ML K. Prostaglandin E(2) is a selective inducer of interleukin-12 p40 (IL-12p40) production and an inhibitor of bioactive IL-12p70 heterodimer. Blood. 2001; 97: 3466-9.

8. M R, M C, V S, F A, A S, GM S, et al. Intestinal immune homeostasis is regulated by the crosstalk between epithelial cells and dendritic cells. Nature immunology. 2005; 6: 507-14.

9. TL D, YC W, SR P, IR W, B P. Lamina propria macrophages and dendritic cells differentially induce regulatory and interleukin 17-producing $\mathrm{T}$ cell responses. Nature immunology. 2007; 8: 1086-94.

10. $\mathrm{S} \mathrm{U}, \mathrm{K} \mathrm{F}, \mathrm{MH} J, \mathrm{BG}$ Y, YJ J, M N, et al. Regulation of humoral and cellular gut immunity by lamina propria dendritic cells expressing Toll-like receptor 5 . Nature immunology. 2008; 9: 769-76.

11. S F, S K, O K, K S. Adaptive immune regulation in the gut: T cell-dependent and $\mathrm{T}$ cell-independent IgA synthesis. Annual review of immunology. 2010; 28: 243-73.

12. JR M, M I, B E, SY S, T J, B S, et al. Generation of gut-homing IgA-secreting B cells by intestinal dendritic cells. Science (New York, NY). 2006; 314: 1157-60.

13. Ma HD, Wang $\mathrm{YH}$, Chang C, Gershwin ME, Lian ZX. The intestinal microbiota and microenvironment in liver. Autoimmunity reviews. 2015; 14: 183-91.

14. Eickmeier I, Seidel D, Grün JR, Derkow $K$, Lehnardt $S$, Kühl AA, et al. Influence of CD8 T cell priming in liver and gut on the enterohepatic circulation. J Hepatol. 2014; 60: 1143-50. 
15. Sipka S, Bruckner G. The immunomodulatory role of bile acids. International archives of allergy and immunology. 2014; 165: 1-8

16. Kabat AM, Srinivasan N, Maloy KJ. Modulation of immune development and function by intestinal microbiota. Trends in immunology. 2014; 35: 507-17.

17. Sommer F, Backhed F. The gut microbiota--masters of host development and physiology. Nature reviews Microbiology. 2013; 11: 227-38.

18. Stary G, Olive A, Radovic-Moreno AF, Gondek D, Alvarez D, Basto PA, et al. VACCINES. A mucosal vaccine against Chlamydia trachomatis generates two waves of protective memory T cells. Science (New York, NY). 2015; 348: aaa8205.

19. DA V, LW C, CJ W. How regulatory $\mathrm{T}$ cells work. Nature reviews Immunology. 2008; 8: 523-32.

20. EM S. Mechanisms of foxp3+ T regulatory cell-mediated suppression. Immunity. 2009; 30: 636-45.

21. VC H, BW H, M BvH, WJ W. The Intestinal Microbiome in Infectious Diseases: The Clinical Relevance of a Rapidly Emerging Field. Open forum infectious diseases. 2017; 4: ofx144.

22. Srivastava A, Gupta J, Kumar S, Kumar A. Gut biofilm forming bacteria in inflammatory bowel disease. Microbial pathogenesis. 2017; 112: 5-14

23. Khan AA, Khan Z, Malik A, Kalam MA, Cash P, Ashraf MT, et al. Colorectal cancer-inflammatory bowel disease nexus and felony of Escherichia coli. Life sciences. 2017; 180: 60-7.

24. Ni J, Wu GD, Albenberg L, Tomov VT. Gut microbiota and IBD: causation or correlation? Nature reviews Gastroenterology \& hepatology. 2017; 14: 573-84.

25. GL S, RD K, A A, HY Z, L S, WH A, et al. Kupffer cell activation by lipopolysaccharide in rats: role for lipopolysaccharide binding protein and toll-like receptor 4. Hepatology (Baltimore, Md). 2000; 31: 932-6.

26. Levy M, Kolodziejczyk AA, Thaiss CA, Elinav E. Dysbiosis and the immune system. Nature reviews Immunology. 2017; 17: 219-32.

27. Pabst $\mathrm{O}$. New concepts in the generation and functions of $\operatorname{IgA}$. Nature reviews Immunology. 2012; 12: 821-32.

28. Mantis NJ, Rol N, Corthesy B. Secretory IgA's complex roles in immunity and mucosal homeostasis in the gut. Mucosal immunology. 2011; 4: 603-11.

29. Mathias A, Pais B, Favre L, Benyacoub J, Corthesy B. Role of secretory IgA in the mucosal sensing of commensal bacteria. Gut microbes. 2014; 5: 688-95.

30. Kim CH. Immune regulation by microbiome metabolites. Immunology. 2018; 154: 220-9.

31. Mowat AM. Anatomical basis of tolerance and immunity to intestinal antigens. Nature reviews Immunology. 2003; 3: 331-41.

32. Lathrop SK, Bloom SM, Rao SM, Nutsch K, Lio CW, Santacruz N, et al. Peripheral education of the immune system by colonic commensal microbiota. Nature. 2011; 478: 250-4

33. Kuhn KA, Stappenbeck TS. Peripheral education of the immune system by the colonic microbiota. Seminars in immunology. 2013; 25: 364-9.

34. Rooks MG, Garrett WS. Gut microbiota, metabolites and host immunity. Nature reviews Immunology. 2016; 16: 341-52.

35. Foey A. Butyrate regulation of distinct macrophage subsets: Opposing effects on M1 and M2 macrophages. International Journal of Probiotics and Prebiotics. 2011; 6: 147-58.

36. N B, S C, Y Z, A W, P P. TLR-2 Signaling Promotes IL-17A Production in CD4+CD25+Foxp3+ Regulatory Cells during Oropharyngeal Candidiasis. Pathogens (Basel, Switzerland). 2015; 4: 90-110.

37. JL R, SM L, J L, G T, B J, TA C, et al. The Toll-like receptor 2 pathway establishes colonization by a commensal of the human microbiota. Science (New York, NY). 2011; 332: 974-7.

38. Ivanov, II, Frutos Rde L, Manel N, Yoshinaga K, Rifkin DB, Sartor RB, et al. Specific microbiota direct the differentiation of IL-17-producing T-helper cells in the mucosa of the small intestine. Cell host \& microbe. 2008; 4: 337-49.

39. Ivanov, II, Atarashi K, Manel N, Brodie EL, Shima T, Karaoz U, et al. Induction of intestinal Th17 cells by segmented filamentous bacteria. Cell. 2009; 139: 485-98.

40. E O, RH H, B M, S M, CK S, JS C. Th17 Cells as Potential Probiotic Therapeutic Targets in Inflammatory Bowel Diseases. International journal of molecular sciences. 2015; 16: 20841-58

41. Bamias G, Cominelli F. Role of type 2 immunity in intestinal inflammation. Current opinion in gastroenterology. 2015; 31: 471-6.

42. Pulendran B, Tang H, Manicassamy S. Programming dendritic cells to induce $\mathrm{T}(\mathrm{H}) 2$ and tolerogenic responses. Nat Immunol. 2010; 11: 647-55.

43. Troy EB, Kasper DL. Beneficial effects of Bacteroides fragilis polysaccharides on the immune system. Frontiers in bioscience (Landmark edition). 2010; 15: $25-34$.

44. Cao Y, Sun Y, Zou S, Duan B, Sun M, Xu X. Yeast beta-Glucan Suppresses the Chronic Inflammation and Improves the Microenvironment in Adipose Tissues of ob/ob Mice. Journal of agricultural and food chemistry. 2018; 66: $621-9$

45. Million M, Tomas J, Wagner C, Lelouard H, Raoult D, Gorvel J-P. New insights in gut microbiota and mucosal immunity of the small intestine. Human Microbiome Journal. 2018; 7-8: 23-32.

46. Tomas J, Wrzosek L, Bouznad N, Bouet S, Mayeur C, Noordine ML, et al. Primocolonization is associated with colonic epithelial maturation during conventionalization. FASEB journal : official publication of the Federation of American Societies for Experimental Biology. 2013; 27: 645-55.

47. Vital M, Howe AC, Tiedje JM. Revealing the bacterial butyrate synthesis pathways by analyzing (meta)genomic data. mBio. 2014; 5: e00889.
48. Kim SH, Cho BH, Kiyono H, Jang YS. Microbiota-derived butyrate suppresses group 3 innate lymphoid cells in terminal ileal Peyer's patches. Scientific reports. 2017; 7: 3980

49. Furusawa Y, Obata Y, Fukuda S, Endo TA, Nakato G, Takahashi D, et al. Commensal microbe-derived butyrate induces the differentiation of colonic regulatory T cells. Nature. 2013; 504: 446-50.

50. Crispe IN. The liver as a lymphoid organ. Annu Rev Immunol. 2009; 27: 147-63.

51. Peng H, Wisse E, Tian Z. Liver natural killer cells: subsets and roles in liver immunity. Cell Mol Immunol. 2016; 13: 328-36.

52. Thakur A, Mikkelsen H, Jungersen G. Intracellular Pathogens: Host Immunity and Microbial Persistence Strategies. Journal of immunology research. 2019; 2019: 1356540

53. Gao B, Jeong WI, Tian Z. Liver: An organ with predominant innate immunity. Hepatology (Baltimore, Md). 2008; 47: 729-36.

54. Dupaul-Chicoine J, Arabzadeh A, Dagenais M, Douglas T, Champagne C, Morizot A, et al. The Nlrp3 Inflammasome Suppresses Colorectal Cancer Metastatic Growth in the Liver by Promoting Natural Killer Cell Tumoricidal Activity. Immunity. 2015; 43: 751-63.

55. Li F, Hao X, Chen Y, Bai L, Gao X, Lian Z, et al. The microbiota maintain homeostasis of liver-resident gammadeltaT-17 cells in a lipid antigen/CD1d-dependent manner. Nature communications. 2017; 7: 13839

56. Wu X, Sun R, Chen Y, Zheng X, Bai L, Lian Z, et al. Oral ampicillin inhibits liver regeneration by breaking hepatic innate immune tolerance normally maintained by gut commensal bacteria. Hepatology (Baltimore, Md). 2015; 62: 253-64.

57. Corbitt N, Kimura S, Isse K, Specht S, Chedwick L, Rosborough BR, et al. Gut bacteria drive Kupffer cell expansion via MAMP-mediated ICAM-1 induction on sinusoidal endothelium and influence preservation-reperfusion injury after orthotopic liver transplantation. Am J Pathol. 2013; 182: 180-91.

58. Wang L, Zhu L, Qin S. Gut Microbiota Modulation on Intestinal Mucosal Adaptive Immunity. Journal of immunology research. 2019; 2019: 4735040.

59. Balmer ML, Slack E, de Gottardi A, Lawson MA, Hapfelmeier S, Miele L, et al. The liver may act as a firewall mediating mutualism between the host and its gut commensal microbiota. Science translational medicine. 2014; 6: 237ra66.

60. Esterházy D, Canesso MCC, Mesin L, Muller PA, de Castro TBR, Lockhart A, et al. Compartmentalized gut lymph node drainage dictates adaptive immune responses. Nature. 2019; 569: 126-30.

61. Kaur D, Patiyal S, Sharma N, Usmani SS, Raghava GPS. PRRDB 2.0: a comprehensive database of pattern-recognition receptors and their ligands. Database (Oxford). 2019; 2019.

62. Paik YH, Schwabe RF, Bataller R, Russo MP, Jobin C, Brenner DA. Toll-like receptor 4 mediates inflammatory signaling by bacterial lipopolysaccharide in human hepatic stellate cells. Hepatology (Baltimore, Md). 2003; 37: 1043-55.

63. Zhu Q, Zou L, Jagavelu K, Simonetto DA, Huebert RC, Jiang ZD, et al. Intestinal decontamination inhibits TLR4 dependent fibronectin-mediated cross-talk between stellate cells and endothelial cells in liver fibrosis in mice. J Hepatol. 2012; 56: 893-9.

64. Iwasaki A, Medzhitov R. Control of adaptive immunity by the innate immune system. Nature immunology. 2015; 16: 343-53.

65. Dapito DH, Mencin A, Gwak G-Y, Pradere J-P, Jang M-K, Mederacke I, et al. Promotion of hepatocellular carcinoma by the intestinal microbiota and TLR4. Cancer cell. 2012; 21: 504-16.

66. Yu L-X, Schwabe RF. The gut microbiome and liver cancer: mechanisms and clinical translation. Nature reviews Gastroenterology \& hepatology. 2017; 14: 527-39.

67. Yu L-X, Yan H-X, Liu Q, Yang W, Wu H-P, Dong W, et al. Endotoxin accumulation prevents carcinogen-induced apoptosis and promotes liver tumorigenesis in rodents. Hepatology (Baltimore, Md). 2010; 52: 1322-33.

68. Yin Z, Ma T, Lin Y, Lu X, Zhang C, Chen S, et al. IL-6/STAT3 pathway intermediates M1/M2 macrophage polarization during the development of hepatocellular carcinoma. Journal of cellular biochemistry. 2018; 119: 9419-32.

69. Miele L, Valenza V, La Torre G, Montalto M, Cammarota G, Ricci R, et al. Increased intestinal permeability and tight junction alterations in nonalcoholic fatty liver disease. Hepatology (Baltimore, Md). 2009; 49: 1877-87.

70. Meroni M, Longo M, Dongiovanni P. Alcohol or Gut Microbiota: Who Is the Guilty? International journal of molecular sciences. 2019; 20

71. Wong J, Johnston B, Lee SS, Bullard DC, Smith CW, Beaudet AL, et al. A minimal role for selectins in the recruitment of leukocytes into the inflamed liver microvasculature. The Journal of clinical investigation. 1997; 99: 2782-90.

72. Lee $W-Y$, Kubes $P$. Leukocyte adhesion in the liver: distinct adhesion paradigm from other organs. Journal of hepatology. 2008; 48: 504-12.

73. Lalor PF, Edwards S, McNab G, Salmi M, Jalkanen S, Adams DH. Vascular adhesion protein-1 mediates adhesion and transmigration of lymphocytes on human hepatic endothelial cells. Journal of immunology (Baltimore, Md : 1950). 2002; 169: 983-92

74. Hintermann E, Christen U. The Many Roles of Cell Adhesion Molecules in Hepatic Fibrosis. Cells. 2019; 8.

75. Borchers AT, Shimoda S, Bowlus C, Keen CL, Gershwin ME. Lymphocyte recruitment and homing to the liver in primary biliary cirrhosis and primary sclerosing cholangitis. Semin Immunopathol. 2009; 31: 309-22.

76. Afford SC, Humphreys EH, Reid DT, Russell CL, Banz VM, Oo Y, et al. Vascular cell adhesion molecule 1 expression by biliary epithelium promotes persistence of inflammation by inhibiting effector T-cell apoptosis. Hepatology (Baltimore, Md). 2014; 59: 1932-43. 
77. Eksteen B, Grant AJ, Miles A, Curbishley SM, Lalor PF, Hübscher SG, et al. Hepatic endothelial CCL25 mediates the recruitment of CCR9+ gut-homing lymphocytes to the liver in primary sclerosing cholangitis. The Journal of experimental medicine. 2004; 200: 1511-7.

78. Trivedi PJ, Bruns T, Ward S, Mai M, Schmidt C, Hirschfield GM, et al. Intestinal CCL25 expression is increased in colitis and correlates with inflammatory activity. Journal of autoimmunity. 2016; 68: 98-104.

79. J W, S C, R C. Immunopathogenesis of primary sclerosing cholangitis. Clinical reviews in allergy \& immunology. 2005; 28: 93-103.

80. Qin N, Yang F, Li A, Prifti E, Chen Y, Shao L, et al. Alterations of the human gut microbiome in liver cirrhosis. Nature. 2014; 513: 59-64.

81. Yan AW, Fouts DE, Brandl J, Stärkel P, Torralba M, Schott E, et al. Enteric dysbiosis associated with a mouse model of alcoholic liver disease. Hepatology (Baltimore, Md). 2011; 53: 96-105.

82. Yoshimoto S, Loo TM, Atarashi K, Kanda H, Sato S, Oyadomari S, et al. Obesity-induced gut microbial metabolite promotes liver cancer through senescence secretome. Nature. 2013; 499: 97-101.

83. Henao-Mejia J, Elinav E, Jin C, Hao L, Mehal WZ, Strowig T, et al, Inflammasome-mediated dysbiosis regulates progression of NAFLD and obesity. Nature. 2012; 482: 179-85.

84. Ponziani FR, Bhoori S, Castelli C, Putignani L, Rivoltini L, Del Chierico F, et al Hepatocellular Carcinoma Is Associated With Gut Microbiota Profile and Inflammation in Nonalcoholic Fatty Liver Disease. Hepatology (Baltimore, Md). 2019; 69: 107-20.

85. Sarin SK, Pande A, Schnabl B. Microbiome as a therapeutic target in alcohol-related liver disease. Journal of hepatology. 2019; 70: 260-72.

86. Saltzman ET, Palacios T, Thomsen M, Vitetta L. Intestinal Microbiome Shifts, Dysbiosis, Inflammation, and Non-alcoholic Fatty Liver Disease. Frontiers in microbiology. 2018; 9: 61.

87. LL Z, X C, PY Z, Y L, GF L, ZQ L, et al. Oral Bifidobacterium modulates intestinal immune inflammation in mice with food allergy. Journal of gastroenterology and hepatology. 2010; 25: 928-34.

88. HL K, PJ VdH, W S, FG P, JP K, ME VdB, et al. Apathogenic, intestinal, segmented, filamentous bacteria stimulate the mucosal immune system of mice. Infection and immunity. 1993; 61: 303-6.

89. GL T, HQ J, NA B, JJ C. Segmented filamentous bacteria are potent stimuli of a physiologically normal state of the murine gut mucosal immune system. Infection and immunity. 1999; 67: 1992-2000.

90. KL F, TL D. Segmented filamentous bacteria-induced immune responses: a balancing act between host protection and autoimmunity. Immunology. 2018.

91. Liwinski T, Zenouzi R, John C, Ehlken H, Rühlemann MC, Bang C, et al. Alterations of the bile microbiome in primary sclerosing cholangitis. Gut. 2019.

92. Palmela C, Peerani F, Castaneda D, Torres J, Itzkowitz SH. Inflammatory Bowel Disease and Primary Sclerosing Cholangitis: A Review of the Phenotype and Associated Specific Features. Gut and liver. 2018; 12: 17-29.

93. Nakamoto N, Sasaki N, Aoki R, Miyamoto K, Suda W, Teratani T, et al. Gut pathobionts underlie intestinal barrier dysfunction and liver $\mathrm{T}$ helper 17 cell immune response in primary sclerosing cholangitis. Nat Microbiol. 2019; 4: 492-503.

94. Frankel AE, Coughlin LA, Kim J, Froehlich TW, Xie Y, Frenkel EP, et al. Metagenomic Shotgun Sequencing and Unbiased Metabolomic Profiling Identify Specific Human Gut Microbiota and Metabolites Associated with Immune Checkpoint Therapy Efficacy in Melanoma Patients. Neoplasia (New York, NY). 2017; 19: 848-55.

95. Routy B, Le Chatelier E, Derosa L, Duong CPM, Alou MT, Daillere R, et al. Gut microbiome influences efficacy of PD-1-based immunotherapy against epithelial tumors. Science (New York, NY). 2018; 359: 91-7.

96. Gopalakrishnan V, Spencer CN, Nezi L, Reuben A, Andrews MC, Karpinets TV, et al. Gut microbiome modulates response to anti-PD-1 immunotherapy in melanoma patients. Science (New York, NY). 2018; 359: 97-103.

97. Matson V, Fessler J, Bao R, Chongsuwat T, Zha Y, Alegre ML, et al. The commensal microbiome is associated with anti-PD-1 efficacy in metastatic melanoma patients. Science (New York, NY). 2018; 359: 104-8.

98. Viaud S, Saccheri F, Mignot G, Yamazaki T, Daillère R, Hannani D, et al. The intestinal microbiota modulates the anticancer immune effects of cyclophosphamide. Science (New York, NY). 2013; 342: 971-6.

99. Zheng Y, Wang T, Tu X, Huang Y, Zhang H, Tan D, et al. Gut microbiome affects the response to anti-PD-1 immunotherapy in patients with hepatocellular carcinoma. Journal for immunotherapy of cancer. 2019; 7: 193.

100. Ma C, Han M, Heinrich B, Fu Q, Zhang Q, Sandhu M, et al. Gut microbiome-mediated bile acid metabolism regulates liver cancer via NKT cells. Science (New York, NY). 2018; 360.

101. Doycheva I, Leise MD, Watt KD. The Intestinal Microbiome and the Liver Transplant Recipient: What We Know and What We Need to Know. Transplantation. 2016; 100: 61-8.

102. Tabibian JH, Kenderian SS. The Microbiome and Immune Regulation After Transplantation. Transplantation. 2017; 101: 56-62.

103. Lu L, Zhou H, Ni M, Wang X, Busuttil R, Kupiec-Weglinski J, et al. Innate Immune Regulations and Liver Ischemia-Reperfusion Injury. Transplantation. 2016; 100: 2601-10.

104. Zhai Y, Petrowsky H, Hong JC, Busuttil RW, Kupiec-Weglinski JW. Ischaemia-reperfusion injury in liver transplantation--from bench to bedside. Nature reviews Gastroenterology \& hepatology. 2013; 10: 79-89.
105. Land WG. The role of postischemic reperfusion injury and other nonantigen-dependent inflammatory pathways in transplantation. Transplantation. 2005; 79: 505-14.

106. Xing HC, Li LJ, Xu KJ, Shen T, Chen YB, Sheng JF, et al. Protective role of supplement with foreign Bifidobacterium and Lactobacillus in experimental hepatic ischemia-reperfusion injury. J Gastroenterol Hepatol. 2006; 21: 647-56.

107. Ren $\mathrm{Z}$, Cui $\mathrm{G}, \mathrm{Lu} \mathrm{H}$, Chen $\mathrm{X}$, Jiang $\mathrm{J}$, Liu $\mathrm{H}$, et al. Liver ischemic preconditioning (IPC) improves intestinal microbiota following liver transplantation in rats through 16s rDNA-based analysis of microbial structure shift. PloS one. 2013; 8: e75950.

108. Qiao YL, Qian JM, Wang FR, Ma ZY, Wang QW. Butyrate protects liver against ischemia reperfusion injury by inhibiting nuclear factor kappa B activation in Kupffer cells. J Surg Res. 2014; 187: 653-9.

109. Yang R, Xu Y, Dai Z, Lin X, Wang H. The Immunologic Role of Gut Microbiota in Patients with Chronic HBV Infection. Journal of immunology research. 2018; 2018: 1-6.

110. Xu D, Huang Y, Wang J. Gut microbiota modulate the immune effect against hepatitis B virus infection. European journal of clinical microbiology \& infectious diseases : official publication of the European Society of Clinical Microbiology. 2015; 34: 2139-47.

111. Wilson ID, Nicholson JK. Gut microbiome interactions with drug metabolism, efficacy, and toxicity. Translational research : the journal of laboratory and clinical medicine. 2017; 179: 204-22.

112. Gong S, Lan T, Zeng L, Luo H, Yang X, Li N, et al. Gut microbiota mediates diurnal variation of acetaminophen induced acute liver injury in mice. Journal of hepatology. 2018; 69: 51-9.

113. Yan AW, Fouts DE, Brandl J, Starkel P, Torralba M, Schott E, et al. Enteric dysbiosis associated with a mouse model of alcoholic liver disease. Hepatology (Baltimore, Md). 2011; 53: 96-105.

114. L W, DE F, P S, P H, P C, C L, et al. Intestinal REG3 Lectins Protect against Alcoholic Steatohepatitis by Reducing Mucosa-Associated Microbiota and Preventing Bacterial Translocation. Cell host \& microbe. 2016; 19: 227-39.

115. Hartmann P, Chen P, Wang HJ, Wang L, McCole DF, Brandl K, et al. Deficiency of intestinal mucin-2 ameliorates experimental alcoholic liver disease in mice. Hepatology (Baltimore, Md). 2013; 58: 108-19.

116. C L, P J, T I, L W, S B, HJ W, et al. Gastric acid suppression promotes alcoholic liver disease by inducing overgrowth of intestinal Enterococcus. Nature communications. 2017; 8: 837 .

117. Y D, C L, S L, K B, H C, L J, et al. Bacteriophage targeting of gut bacterium attenuates alcoholic liver disease. Nature. 2019; 575: 505-11.

118. Knott GJ, Doudna JA. CRISPR-Cas guides the future of genetic engineering. Science (New York, NY). 2018; 361: 866-9.

119. Barrangou R, Notebaart RA. CRISPR-Directed Microbiome Manipulation across the Food Supply Chain. Trends in microbiology. 2019; 27: 489-96. 